\title{
Computational Aspects of Equilibria in Discrete Preference Games
}

\author{
Phani Raj Lolakapuri $^{1 *}$, Umang Bhaskar ${ }^{1}$, Ramasuri Narayanam ${ }^{2}$, Gyana R \\ Parija $^{2}$ and Pankaj S Dayama ${ }^{2}$ \\ ${ }^{1}$ TIFR Mumbai, India \\ ${ }^{2}$ IBM Research, India \\ \{p.lolakapuri, umang\}@tifr.res.in, \{ramasurn, gyana.parija, pankajdayama\}@in.ibm.com
}

\begin{abstract}
We study the complexity of equilibrium computation in discrete preference games. These games were introduced by Chierichetti, Kleinberg, and Oren (EC'13, JCSS '18) to model decision-making by agents in a social network that choose a strategy from a finite, discrete set, balancing between their intrinsic preferences for the strategies and their desire to choose a strategy that is 'similar' to their neighbours. There are thus two components: a social network with the agents as vertices, and a metric space of strategies. These games are potential games, and hence pure Nash equilibria exist. Since their introduction, a number of papers have studied various aspects of this model, including the social cost at equilibria, and arrival at a consensus. We show that in general, equilibrium computation in discrete preference games is PLS-complete, even in the simple case where each agent has a constant number of neighbours. If the edges in the social network are weighted, then the problem is PLScomplete even if each agent has a constant number of neighbours, the metric space has constant size, and every pair of strategies is at distance 1 or 2 . Further, if the social network is directed, modelling asymmetric influence, an equilibrium may not even exist. On the positive side, we show that if the metric space is a tree metric, or is the product of path metrics, then the equilibrium can be computed in polynomial time.
\end{abstract}

\section{Introduction}

Networks are a growing presence in our lives, and affect our behaviour in complex ways. A large amount of literature attempts to understand various facets of these networks. The literature is diverse, due to the large variety of networks and their myriad effects on our daily lives. Prominent among these is the work on opinion formation in social networks [Bala and Goyal, 1998; Golub and Jackson, 2010]; algorithms to target agents in a network to promote adoption of a product [Domingos and Richardson, 2001; Kempe

\footnotetext{
${ }^{*}$ Contact Author
}

et al., 2015]; and models that accurately capture the special structure of social networks [Barabási and Albert, 1999; Watts and Strogatz, 1998].

We study a model of opinion formation in social networks. In a basic but commonly studied model of opinion formation, each agent in the network holds a real-valued opinion, such as her political leaning, and is influenced by her neighbours in the social network. Under the influence of her neighbours, in each time step she updates her opinion to the weighted average of her opinion and that of her neighbours. In a gametheoretic setting, this is a coordination game, where players try to coordinate their opinion with their neighbours. Probabilistic models of updation, where the opinions are from the discrete set $\{0,1\}$ are also studied [Clifford and Sudbury, 1973]. Much of the work in opinion formation focuses on conditions for consensus, when all agents eventually hold the same opinion (e.g., [Acemoglu et al., 2011; Bala and Goyal, 1998]). Clearly, however, consensus is not always attained in social networks, and the basic model has been extended in different ways to capture this lack of consensus (e.g., [Yildiz et al., 2013; Apt et al., 2015]).

Further, most work focuses on the case where the opinion of an agent is either binary (in the set $\{0,1\}$ ), or in the interval $[0,1]$. These are clearly important, since opinions in many cases (e.g., political leanings) are captured by these sets. However, often more complex sets are required. A person's political leaning is often a composite of her inclinations on various topics, such as economic inequality, foreign policy, and taxes. A more realistic model would consider a person's opinion as a composite of these individual opinions. As a second example, a person's opinion could be a physical location, such as a choice of which neighbourhood to live in. Another example would be choosing a technology platform such as Android, iOS, Blackberry, etc. The set of strategies are now discrete points, with distances corresponding to the cost of switching from one technology platform to another.

We study a particular model for opinion formation called a discrete preference game [Chierichetti et al., 2013; Chierichetti et al., 2018]. ${ }^{1}$ In this model, an agent can hold one of a finite set of strategies (opinions), and a distance func-

\footnotetext{
${ }^{1}$ A similar model was concurrently studied by Ferraioli et al. [Ferraioli et al., 2016], however with binary strategies. Both these papers give a natural polynomial time algorithm for equilibrium computation with binary strategies.
} 
tion gives the distance between any pair of strategies. A natural restriction on the distance function is that it be a metric, and hence the strategies are points in a metric space. In addition, each agent has an intrinsic preferred strategy which is fixed. The cost of each agent for a strategy is the sum of weighted distances to her neighbours and to her preferred strategy. The presence of preferred strategies leads to the absence of consensus as an equilibrium in general [Krackhardt, 2009]. Further, the representation of strategies as points in a metric space allows modelling of many complex situations, beyond the simple settings studied earlier.

Since their introduction, numerous papers have studied various properties of these games, including bounding the ratio of the total cost of equilibria to the minimum total cost (called the Price of Anarchy or Stability), as well as generalisations [Auletta et al., 2016; Chierichetti et al., 2018]. In a natural updation process, each player in her turn chooses a strategy that minimizes her cost, given the strategies of the other players. While it is known that this updation process leads to an equilibrium, the number of turns required may be exponential in the size of the game.

In this work, we study computational aspects of equilibria in discrete preference games. Equilibrium computation is a fundamental problem in computational game theory, and the lack of efficient algorithms for this is often viewed as a stumbling block to the notion of equilibria as a prediction of player behaviour (e.g., [Daskalakis et al., 2009]). Algorithms for computing equilibria are also useful, e.g., in simulations to study properties of equilibria, or to obtain approximations to the global optimum for the underlying distance-minimization problem (e.g., [Boykov et al., 2001]).

Coordination games on graphs are another model closely related to discrete preference games [Apt et al., 2017; Apt et al., 2015]. In these games, agents attempt to coordinate with their neighbours, however the set of strategies available to each player is restricted. The distance between any pair of strategies is 1 , and hence these are similar to discrete preference games with the discrete metric.

\subsection{Our Contribution}

We present our results informally here. Formal definitions and results are given in later sections.

We first show that equilibrium computation in discrete preference games is hard, even if we restrict the number of neighbours that each agent has in the social network.

Result 1. It is PLS-hard to find an equilibrium in discrete preference games, even when each player has constant degree in the social network.

If we allow the edges in the social network to be weighted, modelling varying degrees of influence by the neighbours, then equilibrium computation is hard even with multiple restrictions on the metric space.

Result 2. In weighted discrete preference games, it is PLShard to compute an equilibrium, even when each player has constant degree in the social network, the number of strategies is constant, and the distance between any pair of strategies is one or two.
Our results are interesting because these are examples where equilibrium computation is hard in a purely coordination game. In previous games where hardness was shown for equilibrium computation, there were incentives for anti-coordination, i.e., players had an incentive to choose strategies different from their neighbours (e.g., local maxcut games [Schäffer and Yannakakis, 1991], congestion games [Fabrikant et al., 2004], and even coordination-only polymatrix games [Cai and Daskalakis, 2011]).

Lastly, we show that if we allow the edges in the social network to be directed, then an equilibrium may not even exist (and hence, the update process described may cycle).

Result 3. In a discrete preference game with directed edges, an equilibrium may not exist.

We note that directed edges in social networks are clearly more general, and allow the model to capture asymmetric influences. E.g., Facebook offers one the ability to 'follow' another person, which is an asymmetric method of influence. Both undirected and directed social networks are commonly studied (e.g., [Apt et al., 2017; Apt et al., 2015; Bindel et al., 2015; Yildiz et al., 2013]).

In our example to show nonexistence of equilibria, the social network consists of a single strongly connected component. In coordination games on graphs, it is known that if the social network has a single strongly connected component then an equilibrium always exists [Apt et al., 2015]. Our work thus shows this does not hold if we allow more complicated metric spaces.

We show, however, that in two particular cases, an equilibrium can be computed in polynomial time.

Result 4. If the metric space is a tree metric, or is the Cartesian product of path metrics, an equilibrium can be computed in polynomial time.

The case of tree metrics was earlier studied, and bounds shown on the Price of Stability [Chierichetti et al., 2018]. The authors also motivate tree metrics by an example of students choosing a major in college, when different subjects follow a hierarchy for proximity. The product metric space roughly corresponds to the case when the metric space is a regular grid. A natural scenario that is modelled by the product metric is the case presented in the introduction, where an agent's strategy is a composite of a number of individual opinions, and the distance between two strategies is the sum of distances for each individual opinion.

Our algorithms for these cases are simple, however, they obtain equilibria in substantial generalizations of discrete preference games as well, when the social network is a weighted directed graph, and instead of having a single preferred strategy, agents have multiple preferred strategies with different weights for each. Thus, this result also shows the existence of equilibria in directed discrete preference games, with these metric spaces. All missing proofs are given in the full version [Lolakapuri et al., 2019].

\section{Preliminaries}

In the basic model, a discrete preference game consists of an undirected, unweighted neighbourhood graph $G=(V, E)$ 
representing the social network of $n$ players, and a metric space $\mathcal{L}=(L, d)$ [Chierichetti et al., 2018]. Here, $L$ is the set of strategies, and $d$ is a distance metric $-d$ is a function on pairs of strategies that satisfies: (i) $d(x, y) \geq 0$, with equality iff $x=y$, (ii) $d(x, y)=d(y, x)$, and (iii) $d(x, y) \leq d(x, z)+$ $d(y, z)$. Each player $i \in V$ has a preferred strategy $s_{i} \in L$. Since the strategies exist in a metric space, we will also refer to the strategies as points in the metric space. We use $z_{i}$ for the strategy of the $i$ th player, $z=\left(z_{1}, \ldots, z_{n}\right)$ for a strategy profile, and $z_{-i}$ for the strategies of all players except $i$.

Given a parameter $\alpha \in[0,1)$ and a strategy profile $z$, the cost for player $i$ is:

$$
c_{i}(z)=\alpha d\left(s_{i}, z_{i}\right)+(1-\alpha) \sum_{j \in N_{i}} d\left(z_{i}, z_{j}\right),
$$

where $N_{i}$ is the set of neighbours of $i$, not including $i$ herself. Thus the cost of a strategy $z_{i}$ for player $i$ is $\alpha$ times the distance from her preferred strategy, plus $(1-\alpha)$ times the total distance from her neighbours. Each player tries to minimise her cost, and hence tries to choose a strategy that is the weighted median of her preferred strategy and the strategies of her neighbours.

We also study two natural generalisations of the basic model of discrete preference games. The first generalisation allows weights on the edges of the neighbourhood graphs, modelling the scenario when different neighbours of a player have different levels of influence on her. In this case, for player $i$, the strategy profile $z$ has cost:

$$
c_{i}(z)=w_{i} d\left(s_{i}, z_{i}\right)+\sum_{j \in N_{i}} w_{i j} d\left(z_{i}, z_{j}\right),
$$

where $w_{i}$ is the weight player $i$ places on her preferred strategy, and $w_{i j}$ is the weight on the undirected edge $\{i, j\} \in E$.

In the second generalisation, edges are directed as well as weighted, modelling the case when influences are asymmetric. ${ }^{2}$ In this case, the expression for the cost of player $i$ for strategy profile $z$ remains unchanged, though the neighbours of player $i$ are those players that have edges from $i$ in the neighbourhood graph.

An equilibrium is a strategy profile where no player can deviate to a different strategy and reduce her cost. We are interested in algorithms for computing equilibria in discrete preference games. In the undirected weighed setting, these games are exact potential games. That is, for every such game, there is a potential function $\Phi$ of the strategy profile which has the property that if player $i$ deviates from a strategy profile, then the change in player $i$ 's cost is exactly the change in the potential function as well. It can be verified that the potential function for the undirected weighted setting is:

$$
\Phi(z)=\sum_{i \in V} w_{i} d\left(s_{i}, z_{i}\right)+\sum_{\{i, j\} \in E} w_{i j} d\left(z_{i}, z_{j}\right) .
$$

A finite potential game always has an equilibrium, since at the minimum of the potential function, no player has a

\footnotetext{
${ }^{2}$ E.g., Facebook, in addition to the option of adding a person as a friend, offers one the ability to 'follow' another person, which is an asymmetric method of influence.
}

deviating strategy that reduces her cost. Thus, undirected weighted discrete preference games always possess an equilibrium. Further, best response dynamics — where in each step, a player chooses her minimum cost strategy in response to other players, and deviates to it - converges to an equilibrium. However, best-response dynamics may, in general, take exponential time to converge to an equilibrium. In Section 4, we are interested in efficient algorithms for equilibrium computation, that for some polynomial $p(\cdot)$ run in time $O(p(|\mathcal{I}|))$ where $|\mathcal{I}|$ is the size of input, and return an equilibrium.

In Section 3 we show that in general, the problem of equilibrium computation is hard, by showing that even in many simple cases, equilibrium computation is PLS-complete. The class PLS, for Polynomial Local Search, was introduced to study the complexity of finding a local minimum for problems where local search can be carried out in polynomial time [Johnson et al., 1988]. Discrete preference games fall in this class, since finding the equilibrium is equivalent to finding a local minimum for the potential function $\Phi$. The locality of a strategy profile $z$ is the set of all profiles where a single player deviates. By finding the cost of each deviation, for each player, we can obtain a solution with lower value for the potential in polynomial time, if it exists.

A problem is PLS-complete if it is in PLS and is PLShard. PLS-hardness of a problem means that all problems in the class PLS can be polynomially reduced to this problem. Many problems are by now known to be PLS-complete, including local max-cut, max-2SAT, and equilibrium computation in congestion games [Fabrikant et al., 2004; Schäffer and Yannakakis, 1991].

\section{Hardness of Computing Equilibria}

We start with two simple cases when an equilibrium can be computed in polynomial time. Firstly, if the parameter $\alpha \leq 1 / 2$, then in any instance where the neighbourhood graph is connected, the following is an equilibrium: all players choose the same strategy $A \in \mathcal{L}$. If the neighbourhood graph is disconnected, then each isolated player chooses its preferred strategy, while all players in a connected component choose the same strategy. Secondly, in weighted undirected preference games if the weights on the edges as well as the distance between any two strategies are bounded (above and below) by polynomials in the size of the input $\mathcal{I}$, then the equilibrium can be computed in polynomial time by bestresponse dynamics. In this case, the potential is bounded from above by a polynomial in $|\mathcal{I}|$, and in each best-response step, the potential also reduces by a polynomial in $|\mathcal{I}|$. Despite these results, we show that equilibrium computation is in general hard in discrete preference games, even in simple settings. Specifically, we show that in the unweighted setting, for any $\alpha>1 / 2$, computing an equilibrium is PLScomplete even when each player has constant degree. In the weighted setting, computing an equilibrium is PLS-complete even when each player has constant degree, the number of strategies is constant, and the distance between every pair of strategies is either 1 or 2 . For directed neighbourhood graphs, we show that an equilibrium may not even exist.

For the hardness results, we show a reduction from the lo- 


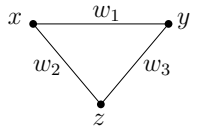

(a)

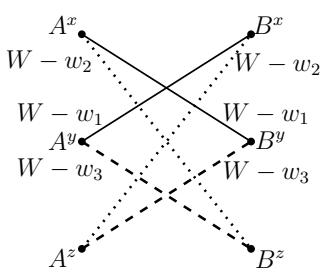

(b)

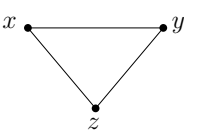

(c)
Figure 1: An example of the reduction from Theorem 1 (in the theorem, each player has five neighbours in the max-cut instance). Figure (a) shows the max-cut instance, (b) shows the metric space in the reduction, and (c) shows the neighbourhood graph. In (b), all pairs of nodes that do not have an edge displayed between them are at distance $W$, where $W=5\left(w_{1}+w_{2}+w_{3}\right)$.

cal max-cut game. In a local max-cut game, we are given an undirected weighted graph with $n$ vertices corresponding to players, and each player has two strategies $A$ and $B$. The utility of a player $i$ is the sum of weights of edges to players that choose the strategy different from $i$, i.e., $u_{i}(z)=$ $\sum_{j \in N_{i}: z_{j} \neq z_{i}} w_{i j}$. Equilibrium computation in the max-cut game is known to be PLS-complete, even if each player has degree five [Elsässer and Tscheuschner, 2011].

Theorem 1. For any $\alpha>1 / 2$ in the unweighted setting, it is $P L S$-hard to find an equilibrium in discrete preference games, even when each player has constant degree.

Proof. Given an instance $G^{\prime}=\left(V^{\prime}, E^{\prime}\right)$ of local max-cut with weights $w^{\prime}$ on the edges, we construct an instance of a discrete preference game where the strategies are in correspondence with the local max-cut game, and in fact the cost in the discrete preference game is exactly a constant minus the utility in the max-cut game. Let $n^{\prime}$ be the number of players in either game, and $W=5 \sum_{e \in E^{\prime}} w_{e}^{\prime}$. We make two assumptions which we remove later: that each player can be restricted to a subset of strategies, and that some players do not have a preferred strategy. With these assumptions, we choose our neighbourhood graph $G(V, E)=G^{\prime}\left(V^{\prime}, E^{\prime}\right)$. The strategy set $L$ contains two strategies $A^{i}$ and $B^{i}$ for each player $i$. We assume that $i$ is restricted to these two strategies. Thus, $|L|=2 n^{\prime}$. Finally, if $\{i, j\} \in E$, then $d\left(A^{i}, B^{j}\right)=d\left(A^{j}, B^{i}\right)$ $=W-w_{i j}$. The distance between any other pair of strategies is $W$. Thus if players $i$ and $j$ both play $A^{i}$ and $A^{j}$, or $B^{i}$ and $B^{j}$, their distance is $W$. Figure 1 shows the reduction for an instance of max-cut with three vertices $x, y$ and $z$.

Note first that the set of players is identical in both games. For every strategy profile $z^{\prime}$ in the max-cut game, there is a strategy profile $z$ in the discrete preference game where player $i$ plays $A^{i}$ if she plays $A$ in the max-cut game, and plays $B^{i}$ otherwise. Then it is easy to see that the cost of player $i$ is $c_{i}(z)=5 W-u_{i}\left(z^{\prime}\right)$. There is thus a correspondence between strategy profiles in the two games, and the cost in one is a constant minus the utility in the other. It follows that $z^{\prime}$ is an equilibrium in the max-cut game iff $z$ (as constructed above) is an equilibrium in the discrete preference game.

We now show how to remove the two assumptions. Our first assumption is that a player can be restricted to two strate- gies. To remove this, for each player $i$, we introduce 20 players: $i_{A, 1}, \ldots, i_{A, 10}$, and $i_{B, 1}, \ldots, i_{B, 10}$. We call these $a u x$ iliary players. Each of these has an edge to player $i$ in the neighbourhood graph, and thus has degree 1. Auxiliary players $i_{A, 1}, \ldots, i_{A, 10}$ have $A^{i}$ as their preferred strategy, while auxiliary players $i_{B, 1}, \ldots, i_{B, 10}$ have $B^{i}$ as their preferred strategy. Since they have degree 1 , and $\alpha>1 / 2$, the best response for these players is always to play their preferred strategy. Now note that since each non-auxiliary player $i$ has degree 25 in the neighbourhood graph, if player $i$ plays either $A^{i}$ or $B^{i}$, her cost is at most $15 W$. However if player $i$ plays a strategy other than $A^{i}$ or $B^{i}$, her cost is at least $20\left(W-\max _{e} w_{e}\right) \geq 16 W$. Hence her best response is always to play either $A^{i}$ or $B^{i}$. Further, since the auxiliary players for player $i$ are equally distributed with $A^{i}$ or $B^{i}$ as the preferred strategy, their addition does not affect player $i$ s choice of strategy between the two, which depends on the strategies chosen by the non-auxiliary players.

Our last assumption is that the non-auxiliary players do not have a preferred strategy. This is removed by introducing another point $C$ into the metric space, which has distance $W$ from all other strategies, and which is the preferred strategy for all non-auxiliary players. However, if $\alpha$ is very large, then it would be an equilibrium for all players to choose $C$. To fix this, increase the number of auxiliary players for each player $i$ from 20 to $\lceil 20 \alpha /(1-\alpha)\rceil$. It can be checked that in this case, player $i$ s best response is always to play either $A^{i}$ or $B^{i}$. We note that each player now has degree at most $\lceil 5+20 \alpha /(1-\alpha)\rceil$, which is a constant for fixed $\alpha$.

The following theorem shows that if the edges in the neighbourhood graph are weighted, equilibrium computation is hard even in simpler settings (even if metric space has only 13 points).

Theorem 2. In the weighted setting, it is PLS-hard to compute an equilibrium, even when each player has constant degree in the neighbourhood graph, the strategy set has constant size, and the distance between any pair of strategies is either one or two.

Proof. As before, given an instance $G^{\prime}=\left(V^{\prime}, E^{\prime}\right)$ of local max-cut with weights $w^{\prime}$ on the edges and degree five for each vertex, we construct an instance of a discrete preference game where the strategy profiles are in correspondence with the local max-cut instance. Let $n^{\prime}=\left|V^{\prime}\right|$ be the number of players and $W=5 \sum_{e \in E^{\prime}} w_{e}^{\prime}$. We describe the reduction under the assumption that each player can choose between two strategies, the assumption can be removed as in Theorem 1. With this assumption, we choose our weighted neighbourhood graph $G(V, E, w)=G^{\prime}\left(V^{\prime}, E^{\prime}, w^{\prime}\right)$.

To construct the metric space, we use the fact that a graph of maximum degree five can be properly coloured by a greedy algorithm with six colours. That is, every vertex in the graph can be assigned one of six colours, so that if vertices $u, v$ are adjacent in the graph, then they are assigned different colours. Thus, the neighbourhood graph can be coloured with six colours. Let $\kappa(v)$ denote the colour assigned to vertex $v \in V$. Let $a, b, c, d, e$, and $f$ be the six colours used. 
Our metric space $\mathcal{L}$ consists of 12 strategies, $\{A, B\} \times$ $\{a, b, c, d, e, f\}$. We call the first component the parity of the strategy, and the second component the colour of the strategy. The distance between two points is 1 if either the parity or colours of the points is different, and is 2 otherwise. We assume that each player $i$ is restricted to the two strategies in the metric space coloured $\kappa(i)$. Note that this means that for a player $i$, since all of her neighbours have a different colour, they cannot be at the same point in the metric space as $i$. Hence the cost of $i$ is at least $\sum_{j \in N_{i}} w_{j}$. Further, it is easily seen that in any strategy profile, the cost of a player $i$ is $2 \sum_{j \in N_{i}} w_{j}$ minus the weight of the neighbours of $i$ that play the parity different from $i$ 's strategy.

For every strategy profile $z^{\prime}$ in the max-cut game, there is a strategy profile $z$ in the discrete preference game where player $i$ plays $(A, \kappa(i))$ if she plays $A$ in the max-cut game, and plays $(B, \kappa(i))$ otherwise. Then the cost of player $i$ in the discrete preference game is $c_{i}(z)=2 \sum_{j \in N_{i}} w_{j}-u_{i}\left(z^{\prime}\right)$. There is thus a correspondence between strategy profiles in the two games, and the cost in one is (a constant plus) the negative of the utility in the other. It follows that $z^{\prime}$ is an equilibrium in the max-cut game if and only if $z$ (as constructed above) is an equilibrium in the discrete preference game.

We now give an example of a directed neighbourhood graph where an equilibrium does not exist. As before, we assume that we can restrict players to a subset of strategies. The assumption can be removed as done in the proof of Theorem 1 , details are given in the full version.

Example 1. With the assumption that we can restrict players to a subset of the strategies, the neighbourhood graph and the metric space for our example are shown in Figure 2. There are three players $x_{0}, x_{1}$, and $x_{2}$. In the neighbourhood graph, player $x_{i}$ has an edge to $x_{i+1} \bmod 3$ (in this example, we always assume $i+1$ is taken mod 3 to avoid repetition). The metric space consists of 6 strategies, $\{0,1,2\} \times\{a, b\}$. We think of the second coordinate as the 'parity', and strategies as nodes in a complete bipartite graph with 6 vertices, with any two strategies of the same parity at distance 2, while any two strategies of different parities at distance 1. By our assumption, we restrict player $x_{i}$ to strategies $(i, a)$ and $(i, b)$.

From the neighbourhood graph, player $x_{i}$ wants to be near player $x_{i+1}$ for $i \in\{0,1,2\}$. However, in the metric space, for any (restricted) choice of strategy for $x_{i+1}$, the strategy of $x_{i}$ that is nearest has the opposite parity. Hence each player $x_{i}$ tries to choose a strategy of the opposite parity from player $x_{i+1}$, and hence there is no equilibrium.

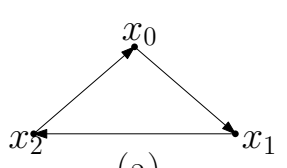

(a)

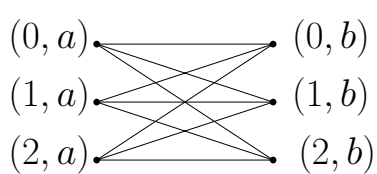

(b)
Figure 2: The example for non-existence of equilibria in directed discrete preference games. Figure (a) shows the neighbourhood graph and (b) shows the metric space. Each edge has unit length.

\section{Algorithms for Computing Equilibria}

We now give efficient algorithms for computing equilibria in discrete preference games with restrictions on the metric space. However, we allow a significant generalization of the neighbourhood graph. We allow directed, weighted neighbourhood graphs, where instead of a preferred strategy, players have a penalty associated with each point in the metric space. Formally, for each node $v$ in the metric space and each player $i$, there is a real-valued penalty $p_{i}(v)$. The cost for player $i$ for the strategy profile $z=\left(z_{i}, z_{-i}\right)$ is

$$
c_{i}(z)=\sum_{v \in L} p_{i}(v) d\left(v, z_{i}\right)+\sum_{j \in N_{i}} w_{i j} d\left(z_{i}, z_{j}\right) .
$$

Our results thus show that in the metric spaces discussed below, equilibria exist, even in the case of directed neighbourhood graphs. E.g., this shows that equilibria exist in the case of path metrics.

We discuss metric spaces in more detail now. Any undirected weighted graph on $r$ vertices corresponds to a metric space with $r$ points, where every vertex is a point, and the distance between any pair of points is the weight of the minimum weight path in the graph between the corresponding vertices. Such a metric space is a graph metric. Further, any finite metric space on $r$ points can be represented as a graph metric, by considering the complete graph on $r$ vertices where the weight of the edge between any pair of vertices is the distance between them.

We first give an algorithm for when the graph metric is a tree, with positive lengths $l_{e}$ on the edges. Note that this contains the special case when the graph metric is a path. We then generalise path metrics in another direction, by considering the Cartesian product of path metrics. This product metric intuitively is obtained when the graph for the metric space is a regular grid.

\subsection{An Algorithm for Tree Metrics}

Our algorithm for tree metrics initially places all players at the root. If any player can improve her cost by moving to a child of her current strategy, the algorithm changes her strategy accordingly. In a metric space with $n$ points, the algorithm terminates in $p n$ iterations of the while loop where $p$ is the number of points, and hence terminates in polynomial time. We now show that when it terminates, the strategy profile is an equilibrium.

To prove convergence, we first characterise the best response. Fix a player $i$ and strategies $z_{-i}$ for the other players. For any node $v$ in the tree, let $w(v)$ be the weight of $i$ 's neighbours $j \in N_{i}$ that have $z_{j}=v$, plus $i$ 's penalty $p_{i}(v)$ for point $v$. This gives us a tree $T$ with weights on the nodes. We say

\footnotetext{
Algorithm 1 Tree Metric Algo

Require: Discrete preference game $(G=(V, E, w), \mathcal{T}=$ $(L, d))$ where $\mathcal{T}$ is a tree metric with root $r$.

1: Initially, let $z_{i} \leftarrow r$ for each player $i$.

2: while $\exists$ player $i$ that can reduce her cost by moving to a child $v$ of $z_{i}$ do $z_{i} \leftarrow v$.
} 
that cost of node $u$ in the tree is the total weighted distance to the other nodes, i.e., $c(u)=\sum_{v \in T} w(v) d(u, v)$. The set of minimum cost nodes in the weighted tree are called the medians of the tree, and are the best responses for player $i$, since $c(u)=c_{i}(z)$ if $z_{i}=u$.

We will use the following result which further characterises the medians. Given weights $w(v)$ at the nodes, let $w(T)$ be the total weight at the nodes of the tree, and $T-v$ be the graph obtained by removing node $v$.

Claim 1 ([Chierichetti et al., 2018]). A node $u$ is the median of a tree iff the weight of each connected component of $T-v$ is at most $w(T) / 2$.

We also use the following claim.

Claim 2. Given a tree $T$ with weights at the nodes, let $v$ be an arbitrary vertex and $v^{*}$ be a median nearest to $v$. Then the cost of the nodes strictly decreases on the path from $v$ to $v^{*}$.

Theorem 3. The Tree Metric Algo terminates at an equilibrium.

Proof. Let $z$ be the strategy profile when the algorithm terminates. Suppose for a contradiction that for player $i, z_{i}=v$, while $z_{i}^{\prime}=v^{*}$ is a nearest best response (and so a median) with lower cost. In the following, we consider the weighted tree $T$ with edge lengths as in the metric space, and weights on the nodes, where for any node $v$ in the tree the weight $w(v)=\sum_{j \in N_{i}: z_{j}=v} w_{i j}+p_{i}(v)$. As earlier, the cost of a node $v$ in the tree is the total weighted distance to the other nodes.

Let $\left(v^{*}=v_{0}, v_{1}, \ldots, v_{t}=v\right)$ be the path from $v^{*}$ to $v$, then by Claim 2, the cost strictly increases along this path, and $c\left(v_{t-1}\right)<c(v)$. Since the algorithm terminates, $v_{t-1}$ must be $v$ 's parent, hence $v \neq r$. Let $T(v)$ be the subtree rooted at $v$. Consider the timestep when player $i$ moved from $v_{t-1}$ to $v$. Note that this decreases $i$ 's distance from every node in $T(v)$ by $l_{v_{t-1}, v}$, and increases the distance from every other node by the same length. Since this move decreased $i$ 's cost, at that time, the total weight of $i$ 's neighbours in $T(v)$ must have been at least $w(T) / 2$. Since that time step, players have only moved away from the root, and hence in particular any player that was in $T(v)$ at that timestep must still be in $T(v)$, and hence when the algorithm terminates, the weight of $i$ 's neighbours in $T(v)$ must be at least $w(T) / 2$. However, since $v^{*}$ is a median, the weight of $i$ 's neighbours in $T(v)$ is also at most $w(T) / 2$. Thus $v$ must also be a median, giving us a contradiction.

\subsection{An Algorithm for the Cartesian Product of Path Metrics}

We now give an algorithm for equilibrium computation if the metric space is the Cartesian product of path metrics. As discussed, a path metric $\mathcal{P}=(L, d)$ can be represented as a path. Alternatively, a path metric can be embedded in the real number line so that the distance between two points is the absolute difference in their values of their embedding.

A metric space $\mathcal{P}=(L, d)$ is the Cartesian product of path metrics $\mathcal{P}_{1}=\left(L_{1}, d_{1}\right), \ldots, \mathcal{P}_{r}=\left(L_{r}, d_{r}\right)$ (or a product metric, for brevity) if $L=L_{1} \times \ldots \times L_{r}$, and for any two points $x=\left(x_{1}, \ldots, x_{r}\right)$ and $y=\left(y_{1}, \ldots, y_{r}\right)$ in $L$,

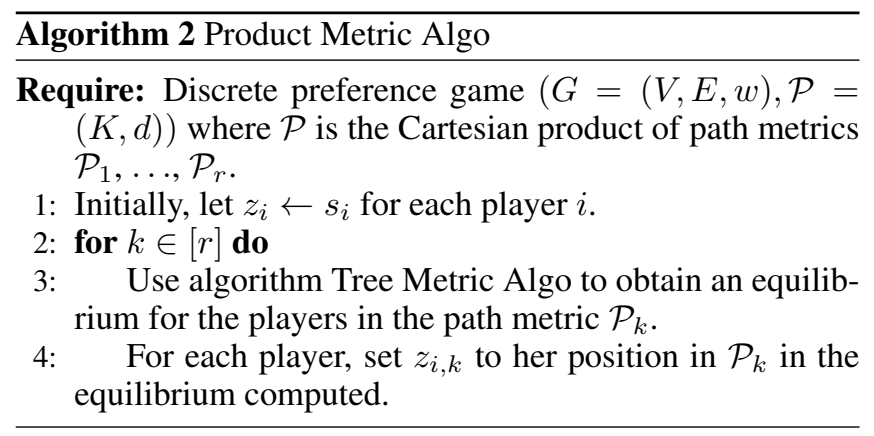

the distance $d(x, y)=\sum_{i=1}^{r} d_{i}\left(x_{i}, y_{i}\right)$. Alternatively, $\mathcal{P}$ is the Cartesian product of $r$ path metrics if it can be embedded in $\mathbb{R}^{r}$, so that the distance between any two points is the $L_{1}$ distance of their embeddings, and whenever $\left(x_{1}, \ldots, x_{r}\right)$ and $\left(y_{1}, \ldots, y_{r}\right)$ are points in the embedding, so are the $2^{r}$ $\left\{x_{1}, y_{1}\right\} \times \ldots \times\left\{x_{r}, y_{r}\right\}$.

For a discrete preference game on a product metric, for each player $i$, her strategy $z_{i}$ is a vector, with the $t$ th coordinate $z_{i, t}$ denoting her position in the path metric $\mathcal{P}_{t}$.

For the algorithm, we first characterize equilibria. Given a discrete preference game with product metric $\mathcal{P}$ and a strategy profile $z$, we say player $i$ is playing her partial best response in the th metric if she is at a median in the path metric $\mathcal{P}_{t}$ (we defined the set of medians earlier, for tree metrics). Note that a player may have multiple best responses.

Claim 3. Player $i$ is playing her best response iff she is playing her partial best response in each metric $t \in[r]$.

Since the Tree Metric Algo terminates in polynomial time, so does the Product Metric Algo.

Theorem 4. The Product Metric Algo terminates at an equilibrium.

\section{Conclusion}

Our work is the first to study the basic question of efficient equilibrium computation in discrete preference games. We show that despite incentivizing coordination, in general equilibrium computation is PLS-hard. However with restrictions on the metric space, equilibrium may be computed in polynomial time, even in very general settings for the neighbourhood graph. Our work is a first step, and leaves open many interesting problems. E.g., for what other metric spaces can we find an equilibrium efficiently? Are there practical restrictions on the neighbourhood graph that make equilibrium computation easier? With the growing popularity of this and other models of opinion formation, we feel these are important, fundamental questions.

\section{Acknowledgements}

Part of this work was done while the first author was an intern at IBM Research, India. Work funded in part by an OSCP joint project between IBM Research, India and TIFR, and a Ramanujan award and an Early Career Research award of the second author. We also thank Harit Vishwakarma and Rakesh Pimplikar for interesting discussions at the initial stages of this project. 


\section{References}

[Acemoglu et al., 2011] Daron Acemoglu, Munther A Dahleh, Ilan Lobel, and Asuman Ozdaglar. Bayesian learning in social networks. The Review of Economic Studies, 78(4):1201-1236, 2011.

[Apt et al., 2015] Krzysztof R. Apt, Sunil Simon, and Dominik Wojtczak. Coordination games on directed graphs. In Proceedings Fifteenth Conference on Theoretical Aspects of Rationality and Knowledge, TARK 2015, Carnegie Mellon University, Pittsburgh, USA, June 4-6, 2015., pages 67-80, 2015.

[Apt et al., 2017] Krzysztof R. Apt, Bart de Keijzer, Mona Rahn, Guido Schäfer, and Sunil Simon. Coordination games on graphs. Int. J. Game Theory, 46(3):851-877, 2017.

[Auletta et al., 2016] Vincenzo Auletta, Ioannis Caragiannis, Diodato Ferraioli, Clemente Galdi, and Giuseppe Persiano. Generalized discrete preference games. In Proceedings of the Twenty-Fifth International Joint Conference on Artificial Intelligence, IJCAI 2016, New York, NY, USA, 9-15 July 2016, pages 53-59, 2016.

[Bala and Goyal, 1998] Venkatesh Bala and Sanjeev Goyal. Learning from neighbours. The Review of Economic Studies, 65(3):595-621, 1998.

[Barabási and Albert, 1999] Albert-László Barabási and Réka Albert. Emergence of scaling in random networks. Science, 286(5439):509-512, 1999.

[Bindel et al., 2015] David Bindel, Jon M. Kleinberg, and Sigal Oren. How bad is forming your own opinion? Games and Economic Behavior, 92:248-265, 2015.

[Boykov et al., 2001] Yuri Boykov, Olga Veksler, and Ramin Zabih. Fast approximate energy minimization via graph cuts. IEEE Trans. Pattern Anal. Mach. Intell., 23(11):1222-1239, 2001.

[Cai and Daskalakis, 2011] Yang Cai and Constantinos Daskalakis. On minmax theorems for multiplayer games. In Proceedings of the Twenty-Second Annual ACM-SIAM Symposium on Discrete Algorithms, SODA 2011, San Francisco, California, USA, January 23-25, 2011, pages 217-234, 2011.

[Chierichetti et al., 2013] F. Chierichetti, J. Kleinberg, and S. Oren. On discrete preferences and coordination. In Proceedings of the 14th ACM Conference on Electronic Commerce (ACM EC), pages 233-250, 2013.

[Chierichetti et al., 2018] Flavio Chierichetti, Jon M. Kleinberg, and Sigal Oren. On discrete preferences and coordination. J. Comput. Syst. Sci., 93:11-29, 2018.

[Clifford and Sudbury, 1973] Peter Clifford and Aidan Sudbury. A model for spatial conflict. Biometrika, 60(3):581588, 1973.

[Daskalakis et al., 2009] Constantinos Daskalakis, Paul W. Goldberg, and Christos H. Papadimitriou. The complexity of computing a nash equilibrium. Commun. ACM, 52(2):89-97, 2009.
[Domingos and Richardson, 2001] Pedro Domingos and Matt Richardson. Mining the network value of customers. In Proceedings of the Seventh ACM SIGKDD International Conference on Knowledge Discovery and Data Mining (KDD '01), pages 57-66. ACM, 2001.

[Elsässer and Tscheuschner, 2011] Robert Elsässer and Tobias Tscheuschner. Settling the complexity of local maxcut (almost) completely. In ICALP, 2011.

[Fabrikant et al., 2004] Alex Fabrikant, Christos H. Papadimitriou, and Kunal Talwar. The complexity of pure nash equilibria. In Proceedings of the 36th Annual ACM Symposium on Theory of Computing, Chicago, IL, USA, June 13-16, 2004, pages 604-612, 2004.

[Ferraioli et al., 2016] Diodato Ferraioli, Paul W. Goldberg, and Carmine Ventre. Decentralized dynamics for finite opinion games. Theor. Comput. Sci., 648:96-115, 2016.

[Golub and Jackson, 2010] B. Golub and M.O. Jackson. Naïve learning in social networks: Convergence, influence, and the wisdom of crowds. American Economics Journal: Microeconomics, 2(1):112-149, 2010.

[Johnson et al., 1988] David S. Johnson, Christos H. Papadimitriou, and Mihalis Yannakakis. How easy is local search? J. Comput. Syst. Sci., 37(1):79-100, 1988.

[Kempe et al., 2015] David Kempe, Jon M. Kleinberg, and Éva Tardos. Maximizing the spread of influence through a social network. Theory of Computing, 11:105-147, 2015.

[Krackhardt, 2009] D. Krackhardt. A plunge into networks. Science, 326:47-48, 2009.

[Lolakapuri et al., 2019] Phani Raj Lolakapuri, Umang Bhaskar, Ramasuri Narayanam, Gyana R Parija, and Pankaj S Dayama. Computational aspects of equilibria in discrete preference games. CoRR, abs/1905.11680, 2019.

[Schäffer and Yannakakis, 1991] Alejandro A. Schäffer and Mihalis Yannakakis. Simple local search problems that are hard to solve. SIAM J. Comput., 20(1):56-87, 1991.

[Watts and Strogatz, 1998] Duncan J Watts and Steven H Strogatz. Collective dynamics of 'small-world' networks. Nature, 393(6684):440, 1998.

[Yildiz et al., 2013] Mehmet Ercan Yildiz, Asuman E. Ozdaglar, Daron Acemoglu, Amin Saberi, and Anna Scaglione. Binary opinion dynamics with stubborn agents. ACM Trans. Economics and Comput, 1(4), 2013. 